\title{
Cytosquelette, phospho-inositides et transduction du signal
}

Des résultats très récents indiquent qu'il existe des relations étroites entre le cytosquelette et le métabolisme des phospho-inositides. Ces connexions - surprenantes et inattendues entre deux familles de molécules aussi différentes - se révèlent particulièrement intéressantes et pourraient jouer un rôle majeur dans la régulation des mécanismes de transduction de signaux extracellulaires et de réorganisation du cytosquelette. L'une des protéines clés qui semble intervenir dans ces mécanismes est la profiline, molécule régulatrice capable de moduler à la fois la polymérisation de l'actine et l'hydrolyse des polyphospho-inositides par la phospholipase $\mathrm{C}_{\gamma} 1$.

\section{Bernard Payrastre}

\section{ADRESSE ET TIRÉS À PART}

B. Payrastre : boursier post-doctoral, fondation pour la recherche médicale. Inserm U. 326, hôpital Purpan, 31059 Toulouse, France. $\mathrm{m} / \mathrm{s} n^{\circ} 2$, vol 8 , février 92 u cours de l'évolution des cellules eucaryotes est apparu un réseau complexe de filaments protéiques appelé cytosquelette (filaments d'actine, microtubules, filaments intermédiaires). De très nombreux secteurs de recherche s'intéressent plus ou moins directement au cytosquelette, son importance dans divers mécanismes cellulaires justifie cet intérêt. Il est bien admis que le maintien de l'intégrité structurale de la membrane plasmique et par là même de la forme des cellules est fortement dépendante des activités du cytosquelette. La polymérisation d'actine est impliquée dans la croissance, les mouvements et la division des cellules. Aujourd'hui, on pense que des protéines étroitement associées au cytosquelette seraient impliquées dans des mécanismes moléculaires ayant lieu à l'interface membrane-cytosol. Ainsi, en plus des protéines spécifiques enchâssées dans la membrane plasmique et servant de centre organisateur pour les réseaux cytosquelettiques [1], des interactions entre des phospholipides membranaires et des protéines capables de se lier à l'actine apparaissent d'une grande importance non seulement pour l'association des filaments cytosquelettiques avec la membrane plasmique, mais aussi pour la régulation de l'organisation du cytosquelette et la régulation de certains mécanismes de transduction du signal. Des résultats récents indiquent en effet que le métabolisme des phospho-inositides (via les récepteurs influençant leur turnover) pourrait être en étroite relation avec l'organisation des filaments d'actine [2, 3, 4]. Il faut également noter que plusieurs des protéines qui 
contrôlent la polymérisation ou la nucléation de l'actine peuvent être activées par des augmentations de concentration de calcium [5].

\section{Interactions phospholipides/ cytosquelette}

\section{RÉFÉRENCES}

1. Carraway KL, Carothers Carraway CA. Membrane-cytoskeleton interactions in animal cells. Biochim Biophys Acta 1989 ; 988 : 147-71.

2. Lassing I, Lindberg U. Specific interaction between phosphatidylinositol 4,5 bisphosphate and profilactin. Nature 1985 : 314: 472-4.

3. Goldschmidt-Clermont PJ, Machesky LM, Baldassare JJ, Pollard TD. The actinbinding protein profilin binds to $\mathrm{PIP}_{2}$ and inhibits its hydrolysis by phospholipase C. Science $1990 ; 247$ : 1575-8.

4. Goldschmidt-Clermont PJ, Kim JW, Machesky LM, Rhec SG, Pollard TD. Regulation of phospholipase C- $\gamma 1$ by profilin and tyrosine phosphorylation. Science $1991 ; 251$ : 1231-3.

5. Forscher P. Calcium and polyphosphoinositide control of cytoskeletal dynamics. Trends Neurol Sci $1989 ; 12$ : 468-74.

6. Isenberg G. Actin binding proteins-lipid interactions. J Muscle Res Cell Motil 1991 ; $12: 136-44$

7. Burn P. Amphitropic proteins : a new class of membrane proteins. Trends Biochem $1988 ; 13: 79-84$.

8. Stossel TP, Chaponnier C, Ezzell RM, et al. Non-muscle actin-binding proteins. Ann Rev Biol 1985 ; 1 : 353-402.

9. Yin HL. Gelsolin : calcium - and polyphosphoinositide - regulated actinmodulating protein. Bio Essays 1988; 7 : 176-9.

10. Janmey PA, Stossel TP. Modulation of gelsolin function by phosphatidylinositol 4,5-bisphosphate. Nature 1987 ; 325 : 362-4.

11. Bryan J. Gelsolin has three actin binding sites. J Cell Biol 1988 ; 106 : 1553-62.

12. Yin $\mathrm{HL}$, Iida $\mathrm{K}$, Janmey PA. Identification of a polyphosphoinositide-modulated domain in gelsolin which binds to the sides of actin filaments. $J$ Cell Biol $1988 ; 106$ : 805-12.

13. Otto JJ. Vinculin. Cell Motil Cytoskel $1990 ; 16: 1-6$

14. Sefton BM, Hunter $\mathrm{T}$, Ball $\mathrm{EH}$, Singer SJ. Vinculin : a cytoskeletal target of the transforming protein of Rous sarcoma virus.
La liste des protéines capables de se lier spécifiquement à l'actine et aux lipides a considérablement augmenté ces dernières années [6]. Malgré la difficulté des études in vivo et les problèmes techniques de relocalisation de certaines protéines après traitement des cellules au Triton X-100, on peut cependant dresser une liste de protéines potentiellement capables de lier l'actine et les phospholipides de façon spécifique et avec une haute affinité (Tableau I). La nature des liaisons non covalentes entre lipides-protéines et protéines-protéines est variée : liaisons hydrogènes, forces de Van der Waals, interactions électrostatiques et hydrophobes [7]. Voici quelques exemples types de ces protéines régulatrices qui contrôlent la polymérisation et/ou se lient à l'actine et aux lipides.

- La gelsoline se lie aux monomères d'actine et peut induire la nucléation de l'actine; elle peut également recouvrir l'extrémité des filaments d'actine [8]. Enfin, la gelsoline peut fragmenter ces filaments de façon dépendante du $\mathrm{Ca}^{2+}$ [9]. Le phosphatidylinositol 4,5-bisphosphate $\left(\mathrm{PIP}_{2}\right)$ et, dans une moindre mesure, le phosphatidylinositol 4-monophosphate (PIP) sont capables d'inhiber de façon spécifique cette fragmentation dépendante du $\mathrm{Ca}^{2+}[9,10]$. La gelsoline possède trois sites de liaison potentiels pour l'actine, un seul serait inhibé par le $\mathrm{PIP}_{2}[11]$. Le domaine de liaison du $\mathrm{PIP}_{2}$ se trouverait dans la région comprise entre les acides aminés 150 et 160 [12], région qui comprend une séquence hydrophobe capable d'interagir avec les chaînes acyl des phospholipides et les résidus basiques. Il est probable que la gelsoline se lie à des agrégats de molécules de $\mathrm{PIP}_{2}$, et l'état physique dans lequel se trouvent les $\mathrm{PIP}_{2}$ et PIP dans la membrane pourrait influencer l'effet inhibiteur. - La vinculine est également une protéine intéressante [13] ; elle joue son rôle au niveau des plaques d'adhérence ou points de contact focaux de cellule à cellule en permettant l'ancrage des microfilaments d'actine à la membrane plasmique. La vinculine est cytosoluble; elle se lie à l'actine et s'insère au domaine hydrophobe de la membrane. Cette insertion pourrait se faire de deux façons : soit par liaisons covalentes avec l'acide myristique ou palmitique, soit par interactions électrostatiques avec des lipides négativement chargés tels que phosphatidylinositol (PI), $\mathrm{PIP}, \mathrm{PIP}_{2}$, phosphatidylsérine (PS), phosphatidylglycérol (PG) et acide phosphatidique (PA). Des modifications minimes, locales et réversibles, dans la composition lipidique de la membrane peuvent moduler ces interactions. La vinculine est phosphorylée par la protéine kinase $\mathrm{C}$ et des tyrosine kinases telles que le produit de l'oncogène src, la protéine pp60 $60^{\text {src }}[14]$. A ce sujet, il est intéressant d'établir une corrélation entre, d'une part, la présence et le rôle de la vinculine au niveau des points de contacts focaux et, d'autre part, le net enrichissement de protéine tyrosine kinases à ce niveau.

- La profiline est une petite protéine basique se liant avec une haute affinité aux monomères d'actine et empêchant ainsi la polymérisation de l'actine [15]. Elle est abondante dans le cytoplasme de la plupart des cellules eucaryotes et a été très étudiée. Lassing et Lindberg [2] ont, les premiers, démontré que le complexe profiline-actine (profilactine) interagissait spécifiquement et avec une forte affinité avec le $\mathrm{PIP}_{2}$ et plus faiblement avec le PIP. De façon tout à fait intéressante, l'interaction $\mathrm{PIP}_{2}$-profiline entraîne la dissociation du complexe profilactine et induit la polymérisation de l'actine in vitro $[2$, 16]. En fait, le $\mathrm{PIP}_{2}$ et l'actine sont en compétition pour le même site de liaison sur la molécule de profiline. Son affinité pour ce phospho-inositide étant plus forte que pour l'actine, lorsque la quantité de $\mathrm{PIP}_{2}$ augmente localement, la dissociation du complexe profilactine peut significativement accroître le taux de monomères d'actine à ce niveau. Ainsi, la fonction de la profiline peut être réglée par le métabolisme des polyphospho-inositides; nous verrons 


\begin{tabular}{|c|c|c|c|c|}
\hline \multicolumn{5}{|c|}{$\begin{array}{l}\text { PRINCIPALES PROTÉINES CONNUES POUR SE LIER À L'ACTINE } \\
\text { ET AYANT UNE AFFINITÉ POUR CERTAINS PHOSPHOLIPIDES ET/OU LE CALCIUM }\end{array}$} \\
\hline Protéines & $\begin{array}{l}\text { P.M. } \\
\text { (kDa) }\end{array}$ & Fonctions & Lipides & $\begin{array}{l}\text { Sensibilité } \\
\text { au } \mathrm{Ca}^{2+}\end{array}$ \\
\hline Myosine I & $110-140$ & Glissement des filaments & $\begin{array}{l}\text { PG, PS, PI, } \\
\text { PIP }_{2}\end{array}$ & \\
\hline Profiline & 15 & Rétention des monomères d'actine & $\mathrm{PIP}_{2}, \mathrm{PIP}$ & \\
\hline $\begin{array}{l}\text { Gelsoline } \\
\text { Villine } \\
\text { Séverine }\end{array}$ & $\begin{array}{l}90 \\
95 \\
40\end{array}$ & $\begin{array}{l}\text { Fragmentation des } \\
\text { filaments et rétention } \\
\text { des monomères d'actine }\end{array}$ & $\begin{array}{l}\mathrm{PIP}_{2}, \mathrm{PIP} \\
? \\
\mathrm{PIP}_{2}, \mathrm{PIP}\end{array}$ & $\begin{array}{l}\text { oui } \\
\text { oui }\end{array}$ \\
\hline Spectrine & $\begin{array}{l}260(\alpha) \\
265(\beta)\end{array}$ & $\begin{array}{l}\text { Ancrage latéral } \\
\text { à la membrane plasmique }\end{array}$ & PS, PG, PE & \\
\hline Taline & 235 & $\begin{array}{l}\text { Ancrage du cytosquelette à la } \\
\text { membrane plasmique, nucléation } \\
\text { d'actine }\end{array}$ & $\begin{array}{l}\text { PG, PS, PC, } \\
\left(P P_{2} \text { ?) }\right.\end{array}$ & \\
\hline $\begin{array}{l}\text { Vinculine } \\
\text { Protéine } 4.1\end{array}$ & $\begin{array}{l}130 \\
78\end{array}$ & $\begin{array}{l}\text { Ancrage du cytosquelette } \\
\text { à la membrane plasmique }\end{array}$ & $\begin{array}{l}\mathrm{PS}, \mathrm{PI}, \mathrm{PG}, \mathrm{PA} \\
\mathrm{PIP}_{2}, \mathrm{PS}\end{array}$ & \\
\hline$\alpha$-actinine & $2 \times 100$ & $\begin{array}{l}\text { Formation des faisceaux de } \\
\text { filaments }\end{array}$ & PA, DG & oui \\
\hline Tropomyosine & $2 \times 35$ & Consolidation des filaments & & oui \\
\hline
\end{tabular}

PG : phosphatidylglycérol ; PS : phosphatidylsérine ; PI : phosphatidylinositol ; PA : acide phosphatidique ; PC : phosphatidylcholine ; DG : diacylglycérol.

que la profiline peut elle-même jouer un rôle régulateur sur ce métabolisme. Cette petite protéine nous permet donc d'aborder le paragraphe suivant dans lequel nous développerons les liens possibles entre le cytosquelette et la transduction du signal.

\section{Implication \\ du cytosquelette dans les mécanismes de transduction du signal}

- Synthèse des polyphospho-inositides et polymérisation de l'actine Dans de nombreux cas, on observe que les agonistes capables de stimuler les cellules en augmentant leur concentration calcique, le métabolisme des phospho-inositides, l'activité de la protéine kinase $\mathrm{C}$, etc., entraînent aussi, très rapidement, une réorganisation du cytosquelette et une polymérisation de l'actine. L'intervention des protéines régulatrices $m / s n^{\circ} 2$, vol. 8, février 92 décrites précédemment est envisagée. Il a été suggéré que les $\mathrm{PIP}_{2}$ étaient potentiellement capables d'entraîner la dissociation pratiquement complète du complexe actine-gelsoline, provoquant une rapide polymérisation de l'actine [5, 17]. Cependant cette suggestion n'est pas de mise dans tous les cas [18].

$\mathrm{Au}$ sujet de la profiline, Lassing et Lindberg [2, 16] ont émis l'hypothèse, dès 1985, qu'une augmentation de la concentration de $\mathrm{PIP}_{2}$ permettrait une dissociation du complexe profilactine, entraînant une polymérisation de l'actine. Ce même groupe a montré que la synthèse des polyphospho-inositides était augmentée après stimulation des plaquettes par la thrombine avant même que ne soit activée la phospholipase C (PLC) [19]. D'autres agonistes, dans d'autres modèles cellulaires, sont également capables d'augmenter cette synthèse de $\mathrm{PIP}_{2}[20]$. Des agents capables d'inhiber la formation et l'hydrolyse des polyphosphoinositides activées par la thrombine réduisent fortement la capacité de cet agoniste à induire la polymérisation de l'actine [21]. Des microdomaines riches en $\mathrm{PIP}_{2}$ peuvent exister, induisant des changements locaux de l'organisation des filaments d'actine. Cependant, Goldschmidt-Clermont et al. [3] ont récemment suggéré que, dans les cellules eucaryotes, la concentration de $\mathrm{PIP}_{2}$ et celle de profiline seraient assez voisines et, vu leur constante de dissociation et le fait que les $\mathrm{PIP}_{2}$ peuvent être agrégés en microdomaines dans la membrane, une grande partie des $\mathrm{PIP}_{2}$ dans la cellule au repos pourrait être complexée avec la profiline. Cette suggestion bouscule un peu l'hypothèse précédente mais permet d'étayer la découverte récente de GoldschmidtClermont et al. [3,4] qui met au jour un nouveau rôle pour les protéines régulatrices de la polymérisation de l'actine et capables de lier les lipides, 
comme nous le décrivons dans le paragraphe suivant.

- Intervention de la profiline dans la régulation de l'activité PLC $\gamma 1$

\section{RÉFÉRENCES}

15. Pollard TD, Cooper JA. Actin and actin-binding proteins. A critical cvaluation of mechanisms and functions. Ann Rev Biochem 1986 ; 55 : 987-1035.

16. Lassing I, Lindberg U. Spccificity of the interaction between phosphatidylinositol 4,5-bisphosphate and the profilin : actin complex. J Cell Biochem $1988 ; 37$ : 255-67.

17. Janmey PA, Stossel TP. Gelsolinpolyphosphoinositide interaction. $J$ Biol Chem 1989 ; 264 : 4825-31.

18. Dadabay CY, Patton E, Cooper JA, Pike L. Lack of correlation between changes in polyphosphoinositide levels and actin/gelsolin complexes in A431 cells treated with cpidermal growth factor. J Cell Biol 1991 ; 112 : 1151-6.

19. I assing I, Lindberg U. Polyphosphoinositide synthesis in platelets stimulated with low concentrations of thrombin is enhanced before the activation of phospholipase C. FEBS Lett 1990 ; 262 : 231-3.

20. Payrastre B, Plantavid M, Breton M Chambaz EM, Chap H. Relationship bet ween phosphoinositide kinase activities and protein tyrosine phosphorylation in plasma membrancs from A431 cells. Biochem $1990: 272: 665-70$.

21. I assing I, Lindberg U. Evidence that the phosphatidylinositide cycle is linked to cell motility. Exp Cell Res 1988 ; 174 : 1-15.

22. Wahl MI, Olashaw NE, Nishibe $\mathrm{S}$ Rhec SG, Pledgder WJ, Carpenter G. Platelet-derived growth factor induces rapid and sustained tyrosinc phosphorylation of phospholipase C- $\gamma$ in quicscent BALB/c3T3 cclls. Mol Cell Biol 1989 : 9 : 2934-43

23. Nishibe S, Wahl MI, HernandezSotomayor SMT, Tonks NK, Rhec SG Carpenter G. Increase of the catalytic activity of phospholipase C- $\gamma 1$ by tyrosine phosphorylation. Science $1990 ; 250$ : 1253-6.

24. Rhei SG. Inositol phosphatidyl-specific phospholipase C : interaction of the $\gamma 1$ isoform with tyrosinc kinase. Trends Biochem $\mathrm{Sci}_{\mathrm{i}}$ $1991 ; 16: 297-301$.

25. Goldschmidt-Clermont PJ, Machesky LM, Doberstein SK, Pollard TD. Mechanims of the interaction of human platelet profilin with actin. $J$ Cell Biol $1991 ; 113$ : 1081-9.

26. Koch AC, Anderson D, Moran MF Ellis C, Dawson T. SH2 and $\mathrm{SH} 3$ domains : clements that control interactions of cytoplasmic signaling protcins. Science $1991 ; 252$ : 668-14
Le facteur de croissance de l'épiderme (EGF), ainsi que le facteur de croissance dérivé des plaquettes (PDGF) sont deux polypeptides capables d'activer par l'intermédiaire de mécanismes de phosphorylation sur des résidus de tyrosine une isoforme cytosolique de la phospholipase C appelée PLC $\gamma 1$, entraînant ainsi la production des scconds messagers inositol trisphosphate et diacylglycérol. Les mécanismes d'activation de la PLC $\gamma 1$ ont été recherchés activement. Il existe une bonne corrélation entrc la phosphorylation sur des résidus de tyrosine de cette enzyme et son activité [22]. Pourtant, l'activité catalytique de la PLC $\gamma 1$ mesurée avec des substrats exogènes n'est pas significativement modifiée par phosphorylation sur des tyrosines (sau dans des conditions particulières en présence d'un détergent [23]). Plusieurs résultats semblent indiquer que l'activation de la PLC $\gamma 1$ par les facteurs de croissance est probablemen due à la levée d'une inhibition (pour une revue récente consulter [24]). Cet inhibiteur pourrait interagir soit avec l'enzyme elle-même, soit avec son substrat. Un candidat potentiel pour jouer ce dernier rôle vient d'être mis en évidence par GoldschmidtClermont et al. [3] qui ont montré que la profiline aurait un rôle inhibiteur pour la PLC $\gamma 1$ en interagissant avec son substrat. En effet, lorsque les molécules de $\mathrm{PIP}_{2}$ sont liécs à la profiline, la PLC $\gamma 1$ non phosphorylée est incapable de les hydrolyser. Cette inhibition est levće dès que la PLC $\gamma 1$ est phosphorylée sur des résidus tyrosine bien précis [4], par exemple, par le réccpteur du PDGF ou de l'EGF. Ainsi, après activation de ces récepteurs par fixation de lcurs agonistes, la PLC $\gamma 1$ est phosphorylée et devient active d'un point de vue enzymatique, même en présence de profiline $\left(\mathrm{m} / \mathrm{s} \quad n^{\circ} 5\right.$, vol. $7, \quad$ p. 513) (figure 1). Après hydrolyse du $\mathrm{PIP}_{2}$, la profiline se retrouve libre pour se lier à l'actine. De façon inattendue, le même groupe a récemment suggéré que la profiline ainsi libérée pourrait avoir un effet transitoire initiateur plutôt qu'inhibiteur de la polymérisation d'actine en augmentant l'échange de nucléotides (ADP/ATP) et la fixation de cations divalents sur l'actine [25].

Cet ensemble de résultats récents demande à être complété et précisé, mais il apparaît que la synthèse du $\mathrm{PIP}_{2}$, son hydrolyse et les complexes profiline-actine ou $\mathrm{PIP}_{2}$-profiline sont des étapes contrôlées qui pourraient joucr un rôle primordial dans les mécanismes de stimulation cellulaire.

- Association de molécules indispensables à la transduction du signal avec le cytosquelette

D'autres résultats intéressants illustrent le rôlc potentiel du cytosquelette dans les mécanismes de transduction du signal.

De nombreuses molécules clés impliquées dans les mécanismes de transduction du signal possèdent en effet un domainc caractérisque appelé $\mathrm{SH} 3$ qui scrait responsable de l'association de ccs protéines avec le cytosquelctte (pour une revue consulter [26]). Nous citerons seulement pour cxemple deux protéines impliquécs dans le métabolisme des phospho-inositides et possédant ce domaine $\mathrm{SH} 3$ non catalytique : la $\mathrm{PLC} \gamma 1$ et la sous-unité $85 \mathrm{kDa}$ de la phosphatidylinositol 3-kinase. Le domaine SH3 pourrait avoir un rôle régulateur dans l'association de ce type d'enzymes avec des éléments du cytosquelette.

Un nombre non négligeable de récepteurs transmembranaires tels que le récepteur de l'EGF [27] ou du facteur de croissance nerveux (NGF) sont également, pour une partie, associés au cytosquelette (Tableau II). La population de récepteurs de l'EGF associée au cytosquelette est fortement enrichie en réceptcurs à haute affinité, la classe de récepteurs responsable de la majeure partie du signal [28], suggérant que cette association joue un rôle important. Une hypothèse attractive est que le cytosquelette jouerait un rôle de coordination en fournissant une matrice sur laquelle diverses composantes des cascades réactionnelles impliquées dans la transduction du signal seraient associées afin de créer un système hautement efficace pour l'activation d'une enzyme par une autre. Nous avons pu récemment montrer une 


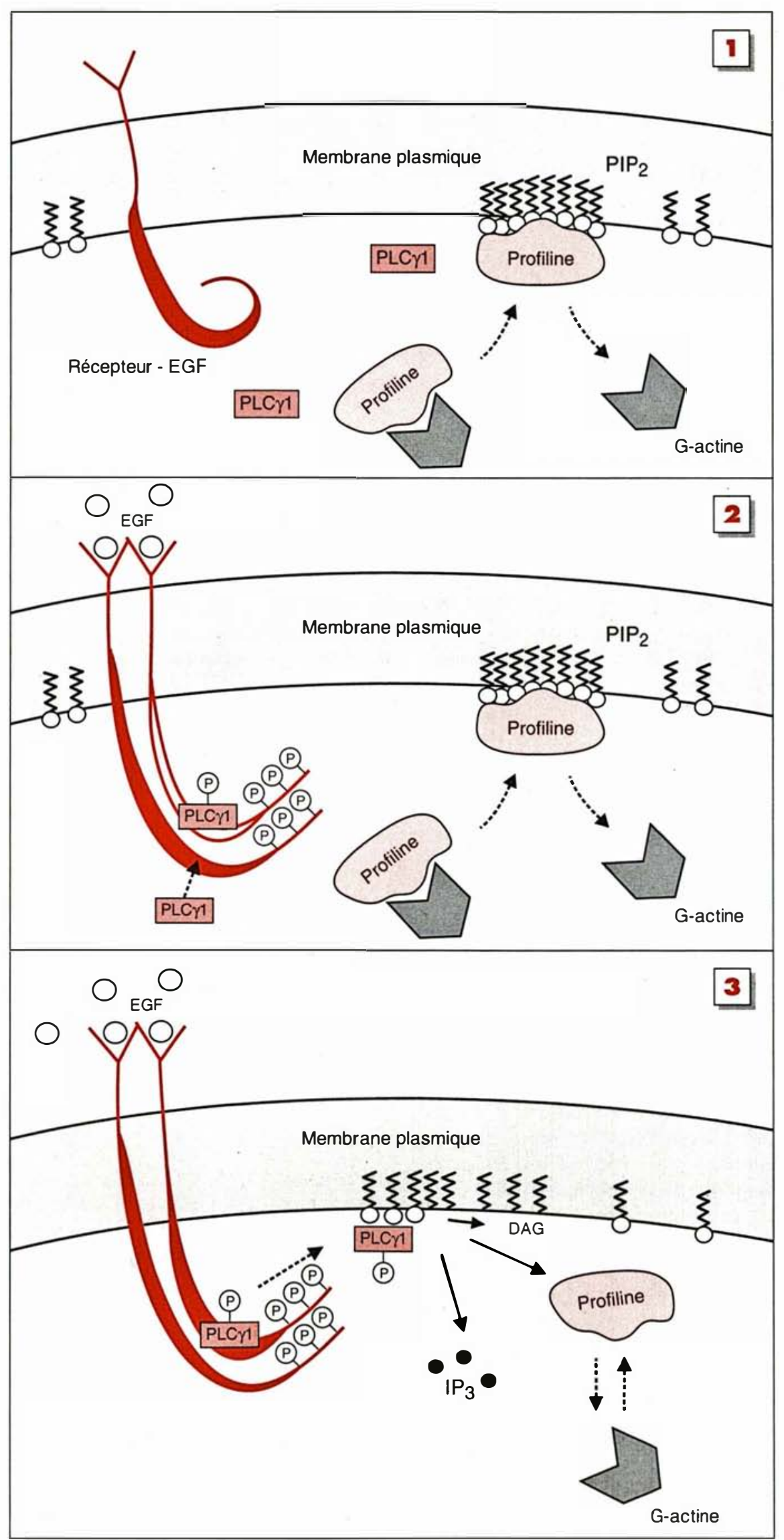

$\mathrm{m} / \mathrm{s} n^{\circ} 2$, vol. 8, féurier 92
Figure 1. Modèle possible de régulation du métabolisme des phospho-inositides et de l'organisation du cytosquelette par la profiline. Lorsque les cellules sont dans un état de repos (1), la profiline pourrait protéger les $\mathrm{PI}, 5 \mathrm{P}_{2}$ de l'hydrolyse par la PLC 1 non phosphorylée. Lors d'une synthèse accrue de $P I 4,5 P_{2}$ l'interaction de haute affinité profilinePI4, $5 P_{2}$ provoquerait une augmentation de la dissociation du complexe profilactine et la libération de G-actine. La liaison de l'EGF à son récepteur (2) induit I'autophosphorylation de ce récepteur et sa dimérisation. La PLC 1 forme alors un complexe avec le récepteur autophosphorylé et est phosphorylée sur les résidus de tyrosine 771, 783 et 1284 par le récepteur de l'EGF activé. L'enzyme phosphorylée est libérée (3) pour éventuellement interagir avec le cytosquelette grâce à son domaine SH3 et devient enzymatiquement active, même sur le complexe P/4,5P -profiline. Cela entraine la production de seconds messagers, inositol trisphosphate (IP $\left.{ }_{3}\right)$ et diacylglycérol (DAG), et la libération de la profiline. La profiline ainsi libérée pourrait avoir un rôle transitoire initiateur plutôt qu'inhibiteur de la polymérisation d'actine [25].

association des enzymes impliquées dans le métabolisme des phosphoinositides avec le cytosquelette. L'activité de ces enzymes est significativement augmentée dans le cytosquelette après stimulation de plaquettes par la thrombine [29] ou de cellules A431 par l'EGF [30].

\section{Conclusion}

De nouvelles notions se dégagent aujourd'hui et indiquent que le cytosquelette peut jouer un rôle essentiel dans les mécanismes directement lićs à la transduction des signaux mitogènes ou hormonaux et à la production de seconds messagers. Nous avons vu ćgalement que plusieurs protéines régulatrices pouvaient non seulement permettre l'ancrage des filaments d'actine à la membrane plasmique grâce à leur interaction avec les phospholipides, mais aussi jouer un rôle dans la rćorganisation du cytosquelette via le contrôle de la polymćrisation d'actine. Ainsi, le métabolisme des polyphospho- 


\begin{tabular}{|c|c|}
\hline \multicolumn{2}{|c|}{$\begin{array}{l}\text { PRINCIPAUX RÉCEPTEURS TRANSMEMBRANAIRES CONNUS } \\
\text { COMME ÉTANT ASSOCIÉS AU CYTOSQUELETTE }\end{array}$} \\
\hline Récepteurs & Références \\
\hline Acétylcholine & $\begin{array}{l}\text { Prives et al. J Cell Biol } 1982: 92: 231-6 \text {; } \\
\text { Stya et Axelrod. J Cell Biol 1983; } 97: \\
48-51 \text {; Bloch J. Cell Biol 1986: } 102: \\
\text { 1447-58. }\end{array}$ \\
\hline EGF & $\begin{array}{l}\text { Landreth et al. J Cell Biol } 1985 ; 101: \\
1341-50 ; \text { Wiegant et al. J Cell Biol } 1986 \text {; } \\
103: 87-94 ; \text { Van Bergen en Henegouwen et } \\
\text { al. J Cell Biochem 1989: } 39: 455-65 .\end{array}$ \\
\hline PDGF & $\begin{array}{l}\text { Zippel et al. Eur J Cell Biol } 1989 \text {; } 50 \text { : } \\
\text { 428-34. }\end{array}$ \\
\hline NGF & $\begin{array}{l}\text { Schechter et Bothwell. Cell } 1981 ; 24: \\
867-74 ; \text { Vale et Shooter. J Cell Biol 1982: } \\
94: 710-7 .\end{array}$ \\
\hline fMLP & $\begin{array}{l}\text { Jesaitis et al. J Cell Biol } 1984 ; 98 \text { : } \\
1378-87 ; \text { Särndhal et al. J Cell Biol } 1989 \text {; } \\
\text { 109: 2791-9. }\end{array}$ \\
\hline Insuline & $\begin{array}{l}\text { Majercik et Bourguignon. Biochem J } 1988 \text {; } \\
252: 815-23 \text {. }\end{array}$ \\
\hline Collagène & $\begin{array}{l}\text { Fox. J Biol Chem } 1985 ; 260: 11970-7 \text {; } \\
\text { Nakano et al. J Biol Chem } 1989 ; 264: \\
5400-6 .\end{array}$ \\
\hline Fibronectine & $\begin{array}{l}\text { Tamkun et al. Cell } 1986: 46: 271-282 \text {; } \\
\text { Buck et Horwitz. J Cell Sci } 1987: 8 \text { (suppl.): } \\
231-50 .\end{array}$ \\
\hline Immunoglobuline $\mathrm{E}$ & Menon et al. J Cell Biol 1986 ; 102 : 541-50. \\
\hline
\end{tabular}

31. Ebcrlc M, Traynor-Kaplan AE, Skla LA, Norgawer J. Is there a relationship between phosphatidylinositol trisphosphate and F-actin polymerization in human ncutrophils? J Biol Chem 1990 ; 265 : 16725-8.

32. Yu FX, Johnston PA, Südhof TC, Yin HL. gCap 39, a calcium - and polyphospoinositide - regulated actin capping protein. Science $1990 ; 250$ : 1413-5

33. Yonczawa $\mathbf{N}$, Nihisda $\mathrm{E}$, Iida $\mathrm{K}$ Yahara I, Sakai H. Inhibition of the interaction of cofilin, destrin and dcoxyribonuclease I with actin by phosphoinositides. $J$ Biol Chem 1990 ; 265 : 8382-6.

34. Yang $Q$, Tonks NK. Isolation of a cDNA clone encoding a human proteintyrosine phosphatase with homology to the cytoskelctal-associated proteins band 4.1 crzin, and talin. Proc Natl Acad Sci USA $1991 ; 88$ : 5949-53.

35. Gu M, York JD, Warshawsky I, Majcrus $\mathrm{PW}$. Identification, cloning and expression of a cytosolic megakaryocyte proteintyrosinc-phosphatase with sequence homology to cytoskcletal protein 4.1. Proc Natl Acad Sci USA 1991; 88 : 5867-71.

36. Vojtck A, Haarer B, Ficld J, et al. Evidence for a functional link between profilin and CAP in the ycast $S$. cerevisiae. Cell 1991 ; 66 : 497-505

37. Goldschmidt-Clermont P, Janmey PA. Profilin, a weak CAP for actin and RAS Cell 1991; 66 : 419-21. inositides et l'organisation des filaments d'actine sont en étroite relation, notamment grâce à une protéine clé : la profiline. De nombreux travaux sont en cours pour préciser ces interactions et gageons qu'une meilleure compréhension de ces mécanismes verra rapidement le jour. Des publications récentes développant de nouvelles hypothèses illustrent l'activité intense de ce secteur de recherche, notamment l'intervention des " nouveaux " phospho-inositides phosphorylés sur la position 3 du D-inositol comme le phosphatidylinositol 3,4,5 trisphosphate $\left(\mathrm{PI} 3,4,5 \mathrm{P}_{3}\right)$ [31], de protéines peu connues comme la gCap 39, qui contrôle la polymérisation de l'actine et se lie au $\mathrm{PIP}_{2}$ [32], ou encore la cofiline et la destrine, qui ont la capacité de se lier aux filaments d'actine et de les dépolymériser de façon dépendante du $\mathrm{pH}$ et qui sont inhibées spécifiquement par les phospho-inositides [33]. Dans ce con- texte, un élément particulièrement intéressant est le clonage des gènes de deux protéine tyrosine phosphatases présentant des homologies avec des protéines du cytosquelette telles que la taline $[34,35]$. Enfin, des résultats tout à fait récents suggèrent une relation fonctionnelle étroite entre la profiline et la protéine CAP (cyclase associated protein) qui appartient au complexe de l'adénylcyclase de Saccharomyces cerevisiae [36, 37]. Cela fournit donc un exemple supplémentaire d'interactions entre le cytosquelette et les voies de signalisation induisant la croissance cellulaire

\section{Remerciements}

Cette revue est l'occasion de remercier les membres du thème 1 de l'Inserm U. 326 : M. Plantavid, G. Mauco, $\mathrm{M}$. Breton et son directeur, le $\mathrm{Pr}$. H. Chap pour leur aide et la relecture de ce manuscrit. 


\section{Summary}

Cytoskeleton, phosphoinositides and signal transduction

Recent papers report interactions between cytoskeleton and phosphoinositide-cycle. These unexpected relationship between two different families of molecules seems to be very important for the control and the modulation of cytoskeleton organization as well as the regulation of signal transduction. Despite the difficulties to study in vivo the associations of proteins to lipids, the list of calcium and phosphoinositides regulated actin modulating protein is rapidly growing. One of the most interesting proteins from this list is the profilin. This protein binds to actin monomers as well as to phosphatidylinositol 4,5-bisphosphate $\left(\mathrm{PIP}_{2}\right)$ and both compete for the same site on the profilin molecule. Since the affinity for $\mathrm{PIP}_{2}$ is higher, the complex profilin-actin can be dissociated by this phospholipid leading to actin polymerization. However, profilin is also able to control the phosphoinositide metabolism. When $\mathrm{PIP}_{2}$ binds to profilin, the unphosphorylated phospholipase $\mathrm{C} \gamma 1$ is unable to hydrolyse $\mathrm{PIP}_{2}$. However, as soon as phospho- lipase $\mathrm{C} \gamma 1$ is phosphorylated on specific tyrosine residues upon growth factor receptor activation, this protein becomes enzymatically active even if $\mathrm{PIP}_{2}$ binds to profilin, leading to the production of the second messengers inositol trisphosphate and diacylglycerol. A few other proteins similar to profilin, like gelsolin or vinculin, are discussed. On the other hand, it is of interest to note that evidence is accumulating that a population of some growth factor receptors is at least partly associated to the cytoskeleton. Also, a number of enzymes from the signal transduction cascade have in common homologous $\mathrm{SH} 3$ sequences. It is suggested that these types of sequences have a regulatory role in the association of proteins to cytoskeletal elements. An attractive hypothesis appears to be a coordinating function of the cytoskeleton. Indeed, cytoskeleton may provide the matrix to which various components of the signal transduction pathway are associated, thus enabling an efficient system for the regulation and the formation of the so-called signal transfer particles.

Jean Hamburger vient de nous quitter. médecine/sciences n'oublie pas la part essentielle prise par Jean Hamburger dans la création et la conception de cette revue. II I'avait souhaitée en langue française, de haute qualité, reconnue internationalement, destinée à mettre en contact la médecine et les sciences biologiques. Toute son œuvre est à l'image de ce projet : faciliter les échanges entre la médecine et les autres sciences du vivant pour mieux comprendre et maîtriser les maladies. Nous avons cherché à être fidèles au vœu de Jean Hamburger et nous n'oublierons pas son message. Que cette revue reste un hommage vivant à sa mémoire. 